\title{
On Burau's representations at roots of unity
}

\author{
Louis Funar \\ Institut Fourier BP 74, UMR 5582 \\ University of Grenoble I \\ 38402 Saint-Martin-d'Hères cedex, France \\ e-mail: louis.funar@ujf-grenoble.fr
}

\author{
Toshitake Kohno \\ Kavli IPMU, Graduate School of Mathematical Sciences \\ The University of Tokyo \\ 3-8-1 Komaba, Meguro-ku, Tokyo 153-8914 Japan \\ e-mail: kohno@ms.u-tokyo.ac.jp
}

February 26, 2013

\begin{abstract}
We consider subgroups of the braid groups which are generated by $n$-th powers of the standard generators and prove that any infinite intersection (with even $n$ ) is trivial. This is motivated by some conjectures of Squier concerning the kernels of Burau's representations of the braid groups at roots of unity. Furthermore, we show that the image of the braid group on 3 strands by these representations is either a finite group, for a few roots of unity, or a finite extension of a triangle group, by using geometric methods.

2000 MSC Classification: 57 M 07, 20 F 36, 20 F 38, 57 N 05.

Keywords: Mapping class group, Dehn twist, Temperley-Lieb algebra, triangle group, braid group, Burau representation.
\end{abstract}

\section{Introduction and statements}

The first part of the present paper is devoted to the study of groups related to the kernels of Burau's representations of the braid groups at roots of unity. We consider two conjectures stated by Squier in [30] concerning these kernels. These conjectures were part of an approach to the faithfulness of Burau's representations and it seems that they were overlooked over the years because of the counterexamples found by Moody, Long, Paton and Bigelow (see $[25,22,4]$ ) for braids on $k \geq 5$ strands.

Specifically, let $B_{k}$ denote the braid group on $k$ strands with the standard generators $g_{1}, g_{2}, \ldots, g_{k-1}$. Squier was interested to compare the kernel of Burau's representation $\beta_{q}$ at a $n$-th root of unity $q$ with the subgroup $B_{k}[n]$ of $B_{k}$ which is the normal closure of the subgroup generated by $g_{j}^{n}$, $1 \leq j \leq k-1$. Our first result answers a strengthened form of the conjecture C2 in [30]:

Theorem 1.1. The intersection of $B_{k}[2 n]$ over any infinite set of integers $n$ is trivial.

Our method does not give any information about the intersection of $B_{k}[n]$ with odd $n$.

The proof uses the asymptotic faithfulness of quantum representations of mapping class groups, due to Andersen ([1]) and independently to Freedman, Walker and Wang ([14]). The other conjecture stated in [30] is that $B_{k}[n]$ is the kernel of Burau's representation. This is false because Burau's representation at a generic parameter is not faithful for $k \geq 5$ (see Proposition 2.4).

The main body of the paper is devoted to the complete description of the image of Burau's representation of $B_{3}$. We can state our main result in this direction as follows:

Theorem 1.2. Assume that $q$ is a primitive $n$-th root of unity, $n \geq 7$ and $g_{1}, g_{2}$ are the standard generators of $B_{3}$. Then $\beta_{-q}\left(B_{3}\right)$ has a presentation with generators $g_{1}, g_{2}$ and relations: 
1. The case $n=2 k$ and $k$ is odd:

$$
\begin{array}{ll}
\text { Braid relation: } & g_{1} g_{2} g_{1}=g_{2} g_{1} g_{2}, \\
\text { Power relations: } & g_{1}^{2 k}=g_{2}^{2 k}=\left(g_{1}^{2} g_{2}^{2}\right)^{k}=1 .
\end{array}
$$

2. The case $n=2 k$ and $k$ is even:

$$
\begin{array}{ll}
\text { Braid relation: } & g_{1} g_{2} g_{1}=g_{2} g_{1} g_{2}, \\
\text { Power relations: } & g_{1}^{2 k}=g_{2}^{2 k}=\left(g_{1}^{2} g_{2}^{2}\right)^{2 k}=1 .
\end{array}
$$

3. The case $n=2 k+1$ :

$$
\begin{array}{ll}
\text { Braid relation: } & g_{1} g_{2} g_{1}=g_{2} g_{1} g_{2}, \\
\text { Power relations: } & g_{1}^{2 k+1}=g_{2}^{2 k+1}=\left(g_{1}^{2} g_{2}^{2}\right)^{2(2 k+1)}=1 .
\end{array}
$$

The remaining cases when $n \in\{1,2,3,4,5,6\}$ are described in Proposition 3.1 and 3.2.

A similar result was announced independently by Masbaum in [23] in a slightly different context. Consider the 2-dimensional $S O(3)$-quantum representations of the mapping class group of the punctured torus at a primitive $2 p$-th root of unity for odd $p$, with the puncture labeled by the color $c=\frac{p-1}{2}-2$. Then the result announced by Masbaum is that the kernel of this representation is normally generated by the $p$-th powers of the Dehn twists. However, these quantum representations are obtained from Burau's representations of $B_{3}$ by quotienting by the (finite) image of the center, so the two results above are equivalent. The same arguments apply to the quantum representations of the mapping class group $M_{0,4}$ of the 4-holed sphere. Notice that 2-dimensional representations of $B_{3}$ are equivalent either to abelian representations, to some not completely reducible representations, or else to Burau's representation.

Another consequence of this theorem is the fact that the image of a pseudo-Anosov mapping class in the mapping class group of the one holed torus by the quantum representations considered above is of infinite order for $p$ large enough. This solves a particular case of a conjecture formulated by Andersen, Masbaum and Ueno in [2]; a proof of the conjecture in this case was announced by Masbaum in [2], Remark 5.9 (see also [16], p.4). We won't give further details since this is also a consequence of a stronger result recently obtained in [29].

The proof of this algebraic statement has a strong geometric flavor. A key ingredient is Squier's theorem concerning the unitarizability of Burau's representation (see [30]). The non-degenerate Hermitian form defined by Squier is invariant under the braid group, but it is not always positive definite. First, we find whether it is positive definite, so that the representation can be conjugate into $U(2)$. On the other hand, when this Hermitian form is not positive definite, the representation can be pseudo-unitarized, namely it can be (rescaled and) conjugated into $U(1,1)$.

We will then focus on the pseudo-unitary case. We show that the image of some free subgroup of the pure braid group $P B_{3}$ on three strands by Burau's representation is a subgroup of $P U(1,1)$ generated by three rotations in the hyperbolic plane. Here, the hyperbolic plane is identified to the unit disk of the complex projective line $\mathbb{C} P^{1}$. Geometric arguments due to Knapp, Mostow and Deraux (see $[19,26,13]$ ) show that the image of $P B_{3}$ is a discrete triangle group and thus we can give an explicit presentation for it. Then an easy argument permits to describe the image of the slightly larger group $B_{3}$. In particular, we obtain a description of the kernel of Burau's representation of $B_{3}$ at roots of unity, which will give a proof of Theorem 1.2.

In a sequel to the present article we will give some applications of these results to the study of the images of the mapping class groups by quantum representations (see [15]). The present paper is 
not only purely technical preparation for the second one. In fact, finding the image of the Burau representation seems to be a difficult problem, which is interesting by itself (see e.g. [8, 7, 24]).

Acknowledgements. We are grateful to Norbert A'Campo, Jørgen Andersen, Jean-Benoît Bost, Martin Deraux, Greg Kuperberg, François Labourie, Yves Laszlo, Greg McShane, Ivan Marin, Gregor Masbaum, Daniel Matei, Majid Narimannejad, Christian Pauly, Bob Penner, Christophe Sorger and Richard Wentworth for useful discussions and to the referees for a careful reading of the paper leading to numerous corrections and suggestions. The first author was partially supported by ANR-06-BLAN-0311 Repsurf and ANR 2011 BS 0102001 ModGroup. The second author is partially supported by Grant-in-Aid for Scientific Research 20340010, Japan Society for Promotion of Science, and by World Premier International Research Center Initiative, MEXT, Japan. A part of this work was accomplished while the second author was staying at Institut Fourier in Grenoble. He would like to thank Institut Fourier for hospitality.

\section{Braid group representations}

\subsection{Jones and Burau's representations at roots of unity}

In this section we recall the definition of the Jones and Burau's representations of the braid groups and show that they are equivalent except at primitive roots of unity of order 1 and 3. Moreover, we discuss when they are unitarizable or pseudo-unitarizable. We start with the following classical definition.

Definition 2.1. The Temperley-Lieb algebra $A_{\tau, k}$, for $\tau \in \mathbb{C}^{*}$ and $k \geq 2$ is the $\mathbb{C}$-algebra generated by the projectors $1, e_{1}, \ldots, e_{k-1}$ satisfying the relations:

$$
\begin{gathered}
e_{j}^{2}=e_{j}, j \in\{1,2, \ldots, k\}, \\
e_{i} e_{j}=e_{j} e_{i}, \text { if }|i-j| \geq 2, \\
e_{j} e_{j+1} e_{j}=e_{j} e_{j-1} e_{j}=\tau e_{j}, \quad j \in\{1,2, \ldots, k\} .
\end{gathered}
$$

There is a natural $\mathbb{C}^{*}$-algebra structure on $A_{\tau, k}$, obtained by setting $e_{j}^{*}=e_{j}, j \in\{1,2, \ldots, k\}$.

According to Wenzl ([31]) there exist such unitary projectors $e_{j}, 1 \leq j \leq k-1$, for any natural number $k \geq 2$ if and only if $\tau^{-1} \geq 4$ or $\tau^{-1}=4 \cos ^{2}\left(\frac{\pi}{n}\right)$, for some natural number $n \geq 3$. However, for given $k$ one could find projectors $e_{1}, \ldots, e_{k-1}$ as above if $\tau^{-1}=4 \cos ^{2}(\alpha)$, where the angle $\alpha$ belongs to some specific arc of the unit circle.

Another definition of the Temperley-Lieb algebra (which is equivalent to the former one, at least when $\tau$ verifies the previous conditions) is as a quotient of the Hecke algebra:

Definition 2.2. The Temperley-Lieb algebra $A_{k}(q)$ is the quotient of the group algebra $\mathbb{C} B_{k}$ of the braid group $B_{k}$ by the relations:

$$
\begin{gathered}
\left(g_{i}-q\right)\left(g_{i}+1\right)=0, \\
1+g_{i}+g_{i+1}+g_{i} g_{i+1}+g_{i+1} g_{i}+g_{i} g_{i+1} g_{i}=0,
\end{gathered}
$$

where $g_{i}$ are the standard generators of the braid group $B_{k}$. The quotient obtained by imposing only the first relation above is called the Hecke algebra $H_{k}(q)$.

It is known that $A_{k}(q)$ is isomorphic to $A_{\tau, k}$ where $\tau^{-1}=2+q+q^{-1}$, and in particular, when $q$ is the root of unity $q=\exp \left(\frac{2 \pi i}{n}\right)$. We suppose from now on that $\tau^{-1}=2+q+q^{-1}$.

We will analyze the case where $k=3$ and $q$ is a root of unity, and more generally for $|q|=1$. Then $A_{\tau, 3}$ and $A_{3}(q)$ are nontrivial and well-defined for all $q$ with $|q|=1$ belonging to the arc 
of circle joining $\exp \left(-\frac{2 \pi i}{3}\right)$ to $\exp \left(\frac{2 \pi i}{3}\right)$ going in counterclockwise direction. We will recover this result below in a slightly different context.

Furthermore, $A_{\tau, 3}$ is semi-simple and splits as $M_{2}(\mathbb{C}) \oplus \mathbb{C}$, where $M_{2}(\mathbb{C})$ denotes the simple $\mathbb{C}$ algebra of 2-by-2 matrices. There is a natural representation of $B_{3}$ into $A_{\tau, 3}$ which sends $g_{i}$ into $q e_{i}-\left(1-e_{i}\right)$. This representation is known to be unitarizable when $\tau^{-1} \geq 4$ (see [17]).

Proposition 2.1. Let $q=\exp (i \alpha)$.

1. Assume that $q$ is not a primitive root of unity of order 2 or 3 . Then every completely reducible representation $\rho$ of $B_{3}$ into $G L(2, \mathbb{C})$ which factors through $A_{3}(q)$ is equivalent to some representation $\rho_{q, C}$ defined by:

$$
\rho_{q, C}\left(g_{1}\right)=\left(\begin{array}{cc}
q & 0 \\
0 & -1
\end{array}\right), \rho_{q, C}\left(g_{2}\right)=\left(\begin{array}{cc}
-\frac{1}{q+1} & -(q+1) C \\
-\epsilon_{q}(q+1) \bar{C} r^{2} & \frac{q^{2}}{q+1}
\end{array}\right),
$$

where $C \in \mathbb{C}-\{0\}, r^{2}=r(q, C)^{2}=|C|^{-2}|q+1|^{-4}|q+\bar{q}+1|$ and $\epsilon_{q}$ is the sign of the real number $q+\bar{q}+1$, namely:

$$
\epsilon_{q}= \begin{cases}1, & \text { if } \alpha \in\left(-\frac{2 \pi}{3}, \frac{2 \pi}{3}\right) \\ -1, & \text { if } \alpha \in\left(\frac{2 \pi}{3}, \pi\right) \cup\left(\pi, \frac{4 \pi}{3}\right)\end{cases}
$$

2. Let $q$ be a primitive root of unity of order 2 or 3 . Then completely reducible representations $\rho$ of $B_{3}$ into $G L(2, \mathbb{C})$ which factor through $A_{3}(q)$ are abelian with finite image and equivalent to:

$$
\rho_{q, 0}\left(g_{1}\right)=\rho_{q, 0}\left(g_{2}\right)=\left(\begin{array}{cc}
q & 0 \\
0 & -1
\end{array}\right) .
$$

We may extend the definition of $\epsilon_{q}, r(q, C)$ to this exceptional case by setting $\epsilon_{q}=1$, if $\alpha \in\left\{-\frac{2 \pi}{3}, \frac{2 \pi}{3}\right\}, \epsilon_{q}=-1$, if $\alpha=\pi$ and $r(q, 0)^{2}=1$. In this case $\rho_{q, 0}$ is both unitarizable and pseudo-unitarizable.

3. If $\alpha \in\left(-\frac{2 \pi}{3}, \frac{2 \pi}{3}\right)$, then the representation $\rho_{q, C}$ is unitarizable if $r(q, C)^{2}=1$.

4. If $\alpha \in\left(\frac{2 \pi}{3}, \frac{4 \pi}{3}\right)$, then the representation $\rho_{q, C}$ is pseudo-unitarizable if $r(q, C)^{2}=1$.

Proof. We can choose $\rho\left(g_{1}\right)=\left(\begin{array}{cc}q & 0 \\ 0 & -1\end{array}\right)$ since completely reducible 2-dimensional representations are diagonalizable and the eigenvalues are prescribed. Since $g_{2}$ is conjugate to $g_{1}$ in $B_{3}$ we have $\rho\left(g_{2}\right)=U \rho\left(g_{1}\right) U^{-1}$, where, without loss of generality, we can suppose that $U \in S L(2, \mathbb{C})$. We discard the case $q=-1$ from now on when the representation should be abelian, as $\rho\left(g_{1}\right)$ is scalar.

Set $U=\left(\begin{array}{ll}a & b \\ c & d\end{array}\right)$, where $a d-b c=1$. Then we have $\rho\left(g_{2}\right)=\left(\begin{array}{cc}q a d+b c & -(q+1) a b \\ (q+1) c d & -q b c-a d\end{array}\right)$. Therefore $\rho$ factors through $A_{3}(q)$, namely the second identity of Definition 2.2 is satisfied, if and only if:

$$
q a d+b c=-\frac{1}{q+1} .
$$

If $1+q+q^{2} \neq 0$, we obtain the solutions: $d=\frac{q}{(q+1)^{2} a}$, and $c=-\frac{q^{2}+q+1}{(q+1)^{2} b}$. This implies that:

$$
\rho\left(g_{2}\right)=\left(\begin{array}{cc}
-\frac{1}{q+1} & -(q+1) C \\
-\frac{\left(q^{2}+q+1\right) q}{(q+1)^{3} C} & \frac{q^{2}}{q+1}
\end{array}\right),
$$

which coincides with the matrix $\rho_{q, C}\left(g_{2}\right)$ in the statement of Proposition 2.1, where $C=a b$ and $r^{2}=r(q, C)^{2}=\frac{|q+\bar{q}+1|}{|q+1|^{4}|C|^{2}}$. 
If $q$ is a primitive root of unity of order 3 , then we find $d=\frac{q}{(q+1)^{2} a}$ and either $b=0$ and $c$ arbitrary or $c=0$ and $b$ arbitrary. But the representation $\rho$ is completely reducible only when $b=c=0$ and this gives the second claim of the Proposition 2.1.

We re-scale the representation $\rho_{q, C}$ so that it takes values in $S L(2, \mathbb{C})$. This amounts to replace $\rho_{q, C}\left(g_{j}\right)$ by $\tilde{\rho}_{q, C}\left(g_{j}\right)=\lambda \rho\left(g_{j}\right)$, where $\lambda$ satisfies $\lambda^{2} q=-1$. Then the condition $r^{2}=1$ is equivalent to $\tilde{\rho}_{q, C}\left(g_{2}\right)=\left(\begin{array}{cc}u & v \\ -\epsilon \bar{v} & \bar{u}\end{array}\right)$, where $|u|^{2}+\epsilon|v|^{2}=1$. In this case the representation $\tilde{\rho}_{q, C}$ takes values in $U(2)$, when $\epsilon=1$ and in $U(1,1)$, when $\epsilon=-1$ respectively.

Remark 2.1. Notice that representations associated to the same $q,|C|^{2}$ are pairwise conjugate.

The representation $\rho_{q, C}$ of $B_{3}$ that arises as above and for which the parameter $C$ satisfies $r(q, C)^{2}=$ 1 will be called the Jones representations of $B_{3}$ at $q$. By the previous remark the conjugacy class of $\rho_{q, C}$ is uniquely determined by the value of $q$. We omit the subscript $C$ in the sequel when the choice of $C$ is not relevant.

Proposition 2.2. Let $\tilde{\rho}: B_{3} \rightarrow S U(2)$ be a unitary Jones representation at $q=\exp (i \alpha)$, for $\alpha \in\left(-\frac{2 \pi}{3}, \frac{2 \pi}{3}\right)$. Let $Q: S U(2) \rightarrow S O(3)$ be the standard double covering map. Then $Q \circ \tilde{\rho}\left(g_{1}\right)$ and $Q \circ \tilde{\rho}\left(g_{2}\right)$ are two rotations of angle $\pi+\alpha$, whose axes form an angle $\theta$ which is given by the formula:

$$
\cos \theta=\frac{\cos \alpha}{1+\cos \alpha} \text {. }
$$

Proof. The set of anti-Hermitian 2-by-2 matrices, namely the matrices $A=\left(\begin{array}{cc}w+i x & y+i z \\ -y+i z & w-i x\end{array}\right)$ with real $w, x, y, z$, is identified with the space $\mathbb{H}$ of quaternions $w+i x+j y+k z$. Under this identification $S U(2)$ corresponds to the sphere consisting of the unit quaternions. In particular, any element of $S U(2)$ acts by conjugacy on $\mathbb{H}$. Let $\mathbb{R}^{3} \subset \mathbb{H}$ be the vector subspace given by the equation $w=0$. Then $\mathbb{R}^{3}$ is $S U(2)$-conjugacy invariant and the linear transformation induced by $A \in S U(2)$ on $\mathbb{R}^{3}$ is the orthogonal matrix $Q(A) \in S O(3)$.

A direct computation shows that $Q\left(\tilde{\rho}\left(g_{1}\right)\right)$ is the rotation of angle $\pi+\alpha$ around the axis $i \in \mathbb{R}^{3}$ in the space of imaginary quaternions. Further $Q\left(\tilde{\rho}\left(g_{2}\right)\right)$ is also a rotation of angle $\pi+\alpha$ since it is conjugate to $Q\left(\tilde{\rho}\left(g_{1}\right)\right)$. As the trace of a rotation of angle $\varphi$ is $1+2 \cos \varphi$ we find the value of $\theta$ as claimed. We omit the details.

Remark 2.2. In [28] the authors consider the structure of groups generated by two rotations of finite order for which axes form an angle which is an integral part of $\pi$. Their result is that there are only few new relations. However, the previous Proposition shows that we cannot apply these results to our situation. It seems quite hard just to find those $\alpha$ for which the axes verify the condition from [28].

Definition 2.3. The (reduced) Burau representation $\beta: B_{k} \rightarrow G L\left(k-1, \mathbb{Z}\left[q, q^{-1}\right]\right)$, for $k \geq 3$, is defined on the standard generators by:

$$
\begin{gathered}
\beta_{q}\left(g_{1}\right)=\left(\begin{array}{cc}
-q & 1 \\
0 & 1
\end{array}\right) \oplus \mathbf{1}_{k-3}, \\
\beta_{q}\left(g_{j}\right)=\mathbf{1}_{j-2} \oplus\left(\begin{array}{ccc}
1 & 0 & 0 \\
q & -q & 1 \\
0 & 0 & 1
\end{array}\right) \oplus \mathbf{1}_{k-j-2}, \text { for } 2 \leq j \leq k-2, \\
\beta_{q}\left(g_{k-1}\right)=\mathbf{1}_{k-3} \oplus\left(\begin{array}{cc}
1 & 0 \\
q & -q
\end{array}\right) .
\end{gathered}
$$


Jones already observed in [17] that the following holds true for the principal roots of unity, i.e., for the roots of unity of the form $\exp \left(\frac{2 \pi i}{n}\right), n \in \mathbb{Z}$ :

Proposition 2.3. Burau's representation of $B_{3}$ at $q$ is conjugate to the tensor product of the parity representation and the Jones representation at $q$, for all $q$ which are not primitive roots of unity of order 2 or 3.

Proof. Recall that the parity representation $\sigma: B_{3} \rightarrow\{-1,1\} \subset \mathbb{C}^{*}$ is given by $\sigma\left(g_{j}\right)=-1$. Burau's representation for $n=3$ is given by

$$
\beta_{q}\left(g_{1}\right)=\left(\begin{array}{cc}
-q & 1 \\
0 & 1
\end{array}\right), \beta_{q}\left(g_{2}\right)=\left(\begin{array}{cc}
1 & 0 \\
q & -q
\end{array}\right) .
$$

Take then $V=\left(\begin{array}{cc}a & \frac{1}{(q+1) a} \\ 0 & \frac{1}{a}\end{array}\right)$, for $q \neq-1$, where $a$ is given by $(q+1)^{3} C a^{2}=1+q+q^{2}$ and $C \neq 0$ is chosen such that $\rho_{q, C}$ is unitarizable, namely $|C|^{2}=|q+1|^{-4}|1+q+\bar{q}|$. One verifies easily that $\left(\sigma \otimes \rho_{q, C}\right)\left(g_{j}\right)=V^{-1} \beta_{q}\left(g_{j}\right) V$.

Remark 2.3. The definition of $A_{\tau, 3}$ in terms of orthogonal projections has a unitary flavor and thus it works properly only when Burau's representation is unitarizable, namely only for those $q=\exp (i \alpha)$, where $\alpha \in\left(-\frac{2 \pi}{3}, \frac{2 \pi}{3}\right)$.

\subsection{Two conjectures of Squier and proof of Theorem 1.1}

This section is devoted to the study of the kernels of the Jones and Burau's representations at roots of unity. Our motivation comes from the following conjectures of Squier in [30]:

Conjecture 2.1 (Squier). The kernel of Burau's representation $\beta_{-q}$ for a primitive $n$-th root of unity $q$ is the normal closure $B_{k}[n]$ of the subgroup of $B_{k}$ generated by $g_{j}^{n}, 1 \leq j \leq k-1$.

The second conjecture of Squier, which is related to the former one, is:

Conjecture 2.2 (Squier). The intersection of $B_{k}[n]$ over all $n$ is trivial.

In order to prove Theorem 1.1, which shows that a stronger version of Conjecture 2.2 holds we will first need a number of definitions and lemmas. Let $D_{k}$ be a disk with $k$ holes. The (pure) mapping class group $M\left(D_{k}\right)$ is the group of framed pure braids $\widetilde{P B}_{k}$ and fits into the exact sequence:

$$
1 \rightarrow \mathbb{Z}^{k} \rightarrow \widetilde{P B}_{k} \rightarrow P B_{k} \rightarrow 1
$$

where $\mathbb{Z}^{k}$ is generated by the Dehn twists along the boundary curves.

The extended mapping class group $M^{*}\left(D_{k}\right)$ is the group of mapping classes of homeomorphisms of the disk with $k$ holes that fix point-wise the boundary of the disk but are allowed to permute the remaining boundary components, which are suitably parameterized. Thus $M^{*}\left(D_{k}\right)$ is the group of framed braids on $k$ strands and we have then the exact sequence:

$$
1 \rightarrow \mathbb{Z}^{k} \rightarrow M^{*}\left(D_{k}\right) \rightarrow B_{k} \rightarrow 1
$$

Since the unit tangent bundle has a section the exact sequence above has a non-canonical splitting, i.e., there exists a section $s: B_{k} \hookrightarrow M^{*}\left(D_{k}\right)$, which we fix once for all. The restriction of $s$ to the subgroup $P B_{k}$ yields a section $P B_{k} \hookrightarrow \widetilde{P B}_{k}$. Let $g_{1}, \ldots, g_{k-1}$ denote the standard generators of $B_{k}$. 
Definition 2.4. Let $n$ be a positive integer. The subgroup $B_{k}\{n\}$ of $B_{k}$ is the normal closure of the subgroup generated by the elements:

$$
g_{1}^{2 n},\left(g_{1} g_{2}\right)^{3 n},\left(g_{1} g_{2} g_{3}\right)^{4 n}, \ldots,\left(g_{1} g_{2} \cdots g_{k-2} g_{k-1}\right)^{k n} .
$$

Observe that $B_{k}[2 n] \subset B_{k}\{n\}$. In fact $B_{k}[2 n]$ is the normal closure of the subgroup of $B_{k}$ generated by the element $g_{1}^{2 n}$, since the standard generators $g_{j}$ are conjugate.

Definition 2.5. For any compact orientable surface $\Sigma$ (possibly with boundary) we set $M(\Sigma)[n]$ for the normal subgroup of $M(\Sigma)$ generated by the $n$-th powers of Dehn twists.

Lemma 2.1. We have $s\left(B_{k}\{n\}\right) \subset M\left(D_{k}\right)[n]$.

Proof. Every element of the given set of normal generators of $B_{k}\{n\}$ is a pure braid and hence $B_{k}\{n\} \subset P B_{k}$. Furthermore, let us observe that $\delta_{j}=\left(g_{1} g_{2} \cdots g_{j-1} g_{j}\right)^{j+1}$, for $j=1,2, \ldots, k-1$, is a Dehn twist along a curve encircling the first $j+1$ punctures of the $k$-punctured disk. Thus $B_{k}\{n\}$ is normally generated by some $n$-th powers of Dehn twists and thus it is contained in the normal closure in $B_{k}$ of the subgroup generated by the $n$-th powers of all Dehn twists.

Conversely, let $\gamma$ be an embedded curve in the $k$-punctured disk which encircles $j+1 \geq 2$ punctures. Then the (right) Dehn twist $T_{\gamma}$ along the curve $\gamma$ is conjugate in $B_{k}$ to $\delta_{j}$ by means of the class of a homeomorphism of the $k$-punctured disk sending $\gamma$ into the curve encircling the first $j+1$ punctures. Thus $B_{k}\{n\}$ contains the subgroup generated by the $n$-th powers of Dehn twists on the $k$-punctured disk. The later is a normal subgroup of $B_{k}$ and hence it coincides with its normal closure. Thus $B_{k}\{n\}$ is the subgroup generated by the $n$-th powers of all Dehn twists on the $k$-punctured disk.

The lift of a Dehn twist $T_{\gamma} \in P B_{k}$ into the mapping class group $M\left(D_{k}\right)$ of the $k$-holed disk is of the form $s\left(T_{\gamma}\right)=T_{\gamma} \prod_{i} T_{c_{i}}^{\varepsilon_{i}}$, where $\varepsilon_{i} \in \mathbb{Z}$ and $T_{c_{i}}$ are Dehn twists along boundary components of $D_{k}$. Therefore $s\left(T_{\gamma}^{k}\right) \in M\left(D_{k}\right)[k]$. This proves the claim.

Remark 2.4. In a similar way we can identify $B_{k}[2 n]$ with the subgroup of $B_{k}$ generated by Dehn twists along curves encircling precisely 2 punctures.

The main result of this section is the following one which implies immediately Theorem 1.1 in the Introduction:

Theorem 2.1. The intersection of $B_{k}\{n\}$ over an infinite set of integers $n$ is trivial.

Before to proceed we need some preliminaries concerning quantum representations. Recall that in [6] the authors defined the TQFT functor $\mathcal{V}_{p}$, for every $p \geq 3$ and a primitive root of unity $A_{p}$. These TQFT should correspond to the so-called $S U(2)$-TQFT, for even $p$ and to the $S O(3)$-TQFT, for odd $p$ (see also [21] for another $S O(3)$-TQFT).

Definition 2.6. Let $p \in \mathbb{Z}_{+}, p \geq 3$, such that $p \not \equiv 2(\bmod 4)$. The quantum representation $\rho_{p}$ is the projective representation of the mapping class group associated to the TQFT $\mathcal{V}_{\frac{p}{2}}$ for even $p$ and $\mathcal{V}_{p}$ for odd $p$, respectively. This amounts to saying that $A_{p}$ is a primitive root of unity of order $p$, when $p$ is even, and of order $2 p$, otherwise.

Consider now the projective quantum representations $\rho_{p}$ of $M_{k+1}$ from Definition 2.6. According to $[1,14]$, for any infinite set of even integers $A$ we have $\cap_{p \in A}$ ker $\rho_{p}=1$. This statement is usually called the asymptotic faithfulness of quantum $S U(2)$-representations. However, the proof given in [14] for the the $S U(2)$-TQFT extends without any essential modification to the $S O(3)$-TQFTs $\mathcal{V}_{p}$ defined in [6]. Therefore the asymptotic faithfulness condition $\cap_{p \in A}$ ker $\rho_{p}=1$, holds now for 
any infinite set of integers $A$, not necessarily consisting of even numbers. Recall also that $\rho_{p}$ was defined in Definition 2.6 only when $p \not \equiv 2(\bmod 4)$.

The eigenvalues of a Dehn twist in the TQFT $\mathcal{V}_{p}$ i.e., the entries of the diagonal $T$-matrix are of the form $\mu_{l}=\left(-A_{p}\right)^{l(l+2)}$, where $l$ belongs to the set of admissible colors (see [6], 4.11). The set of admissible colors in the theory $\mathcal{V}_{\frac{p}{2}}$ is $\left\{0,1,2, \ldots, \frac{p}{2}-2\right\}$, in the theory $\mathcal{V}_{\frac{p}{2}}$ for $p \equiv 0(\bmod 4)$ and $\{0,2,4, \ldots, p-3\}$ in the theory $\mathcal{V}_{p}$, for odd $p$, respectively. Therefore the order of the image of a Dehn twist by $\rho_{p}$ divides $p$. This divisibility property is the reason for defining $\rho_{p}$ for even $p$ as being associated to the TQFT $\mathcal{V}_{\frac{p}{2}}$ instead of $\mathcal{V}_{p}$.

Proof of Theorem 1.1. When $k=2$, the claim holds trivially. Assume henceforth that $k \geq 3$. We embed $D_{k}$ into the closed orientable surface $\Sigma_{k+1}$ of genus $k+1$ by gluing a one-holed torus along each boundary component. Let $M_{k+1}$ denote the mapping class group of $\Sigma_{k+1}$. According to [27] the homomorphism $i: M\left(D_{k}\right) \rightarrow M_{k+1}$ induced by the inclusion of surfaces is injective.

We have obviously $i\left(M\left(D_{k}\right)[n]\right) \subset M_{k+1}[n]$ and so Lemma 2.1 implies that $i\left(s\left(B_{k}\{n\}\right)\right) \subset M_{k+1}[n]$.

Therefore, $M_{k+1}[p] \subset$ ker $\rho_{p}$, for any $p$. Then the asymptotic faithfulness of quantum $S U(2)$ and $S O(3)$-representations and the injectivity of $i \circ s$ imply that $\cap_{p \in A} B_{k}\{p\}=1$, for any infinite set $A$.

Remark 2.5. The weaker statement that $\cap_{n \in \mathbb{Z}-\{0\}} B_{k}[n]=1$ can also be shown by means of the residual finiteness of the braid group. This was independently observed by Ivan Marin. Consider a residually finite group $G$ having a finite system of generators $S$. Let $G[n]$ be the normal closure of the subgroup of $G$ generated by $s^{n}$, with $s \in S$. We claim that $\cap_{n \in \mathbb{Z}-\{0\}} G[n]=1$. In fact, suppose that there exists $1 \neq a \in \cap_{n \in \mathbb{Z}-\{0\}} G[n]$. By the residual finiteness of $G$ there exists some finite group $F$ and a morphism $f: G \rightarrow F$ with $f(a) \neq 1$. Now $f(s)^{n}=1$, for every $s \in S$, where $n$ is the order of the finite group $F$. This shows that $f$ factors through $G / G[n]$, which implies that $f(a)=1$, contradicting our assumption. This proves the claim. In particular, this implies that

$$
\cap_{n \in \mathbb{Z}-\{0\}} B_{k}[n]=\cap_{n \in \mathbb{Z}-\{0\}} B_{k}\{n\}=1, \cap_{n \in \mathbb{Z}-\{0\}} M_{k}[n]=1 .
$$

However, it seems that the proof of the stronger claim of Theorem 2.1 uses in an essential way the asymptotic faithfulness of the quantum representations.

Proposition 2.4. Then Conjecture 2.1 for roots of unity $q$ of even order and given $k \geq 5$ is false for all but finitely many $q$.

Proof. One knows by results of Bigelow ([4]), Moody ([25]), Long and Paton ([22]) that for $k \geq 5$ the (generic i.e., for a formal indeterminate $q$ ) Burau representation $\beta$ into $G L\left(k-1, \mathbb{Z}\left[q, q^{-1}\right]\right.$ ) is not faithful. Let $a \in B_{k}$ be such a non-trivial element in the kernel of $\beta$.

Suppose that Conjecture 2.1 is true for infinitely many primitive roots of unity $q$ of even order. Then $a$ should belong to the intersection of kernels of all $\beta_{q}$, over all roots of unity $q$.

By Theorem 1.1 we have $\cap_{n=2}^{\infty} B_{k}[2 n]=1$. If $\operatorname{ker} \beta_{q}=B_{k}[2 n]$ for infinitely many roots of unity $q$ of even order $2 n$, it follows that $a \in \cap_{n=2}^{\infty} B_{k}[2 n]=1$, which is a contradiction.

Remark 2.6. The TQFT are unitary, namely the representations $\rho_{p}$ are unitary representations, if the choice of the primitive roots of unity $A_{p}$ is the following one:

$$
A_{p}= \begin{cases}-\exp \left(\frac{2 \pi i}{p}\right), & \text { if } p \equiv 0(\bmod 4) ; \\ \exp \left(\frac{\left(p^{2}+1\right) \pi i}{2 p}\right), & \text { if } p \equiv 1(\bmod 2) .\end{cases}
$$

However, the choice of $A_{p}$ does not have any impact on the proof of the Theorem. Although in the proof of the asymptotic faithfulness given in [14] one considers the unitary TQFT, the proof works as well for any other primitive roots of unity, by using a Galois conjugacy. 


\section{The image of Burau's representation of $B_{3}$ at roots of unity}

\subsection{Finite and exceptional quotients of $B_{3}$}

The aim of this section is to understand the image of Burau's representation $\beta_{-q}\left(B_{3}\right)$ at roots of unity of small order and, in particular, to find an explicit presentation of it. Notice that we will consider the representation at the root $-q$, instead of $q$, for reasons that will appear later.

If one is interested to know whether $\beta_{-q}\left(B_{3}\right)$ is discrete one should first analyze the case when the image can be conjugated into $U(2)$, and then re-scale it into $S U(2)$. There the discreteness is equivalent to the finiteness of the image. The finiteness of the Jones representation of $B_{3}$ was completely characterized in [17]. Jones studied the case where the roots of unity $-q$ have the form $-q=\exp \left(\frac{2 \pi i}{k}\right)$, but the Galois conjugation yields isomorphic groups so that the discussion in [17] is complete. The only cases where the image of the Jones representation of $B_{3}$ at $-q$ is finite is when $-q$ is a primitive root of unity of order 1,2,3,4,6 or 10 . Moreover, Burau's representation is equivalent to the Jones representation only when the root of unity is neither -1 nor a primitive third root of unity. These excluded cases should be treated separately. For the sake of completeness we sketch the proofs below.

Proposition 3.1. Let $q$ be a primitive root of unity of order $n \in\{2,3,4,5\}$. Then $\beta_{-q}\left(B_{3}\right)$ is a finite group with the group presentation:

$$
\left\langle g_{1}, g_{2} \mid g_{1} g_{2} g_{1}=g_{2} g_{1} g_{2}, g_{1}^{n}=g_{2}^{n}=1\right\rangle .
$$

Proof. Set $B_{k}(n)=B_{k} / B_{k}[n]$. Then Burau's representation $\beta_{-q}$ factors through $B_{3}(n)$ when $q$ is a primitive root of unity of order $n$.

Coxeter gave in [10] the exhaustive list of the groups $B_{k}(n)$ which are finite, together with their respective description (see also $[11,12]$ ). The finite ones are those for which $(k-2)(n-2)<4$. Namely, when $k=3$, there is the following list:

1. $B_{3}(2)$ is the symmetric group $S_{3}$;

2. $B_{3}(3)$ is isomorphic to $S L(2, \mathbb{Z} / 3 \mathbb{Z})$ (or the binary tetrahedral group $\Delta(2,3,3)$, see section 3.3 for definitions) and has order 24 ;

3. $B_{3}(4)$ is isomorphic to the triangle group $\Delta(2,3,4)$ and has order 96 ;

4. $B_{3}(5)$ is isomorphic to $G L(2, \mathbb{Z} / 5 \mathbb{Z})$ and has order 600 .

Set $N(n) \subset B_{3}(n)$ for the group generated by the image of $\left(g_{1} g_{2}\right)^{3}$, which is a generator of the center of $B_{3}$. By a direct computation we show that $\beta_{-q}\left(\left(g_{1} g_{2}\right)^{3}\right)=-q^{3} \mathbf{1}$ is a scalar matrix and thus $\beta_{-q}$ induces a well-defined homomorphism $\tilde{\beta}_{-q}: B_{3}(n) / N(n) \rightarrow P G L(2, \mathbb{C})$. Furthermore, we have the following commutative diagram:

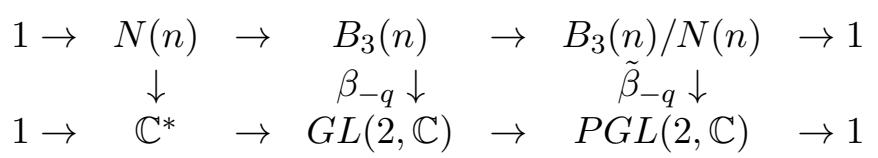

Further, one verifies that $\beta_{-q}\left(\left(g_{1} g_{2}\right)^{3}\right)=-q^{3} 1$ has order $o(n)$, where $o(2)=1, o(3)=2, o(4)=$ $4, o(5)=10$. Since the order of $N(n)$ is also $o(n)$ it follows that the restriction of $\beta_{-q}$ at $N(n)$ is injective. 
From the previously cited results of Coxeter we derive that:

$$
B_{3}(n) / N(n)= \begin{cases}S_{3}, & \text { if } n=2 \\ A_{4}, & \text { if } n=3 \\ S_{4}, & \text { if } n=4 \\ A_{5}, & \text { if } n=5\end{cases}
$$

where $S_{m}$ and $A_{m}$ denote the symmetric and the alternating group on $m$ elements, respectively.

A direct inspection shows that the image of $\tilde{\beta}_{-q}$ is non-abelian because $\tilde{\beta}_{-q}\left(g_{1} g_{2}\right) \neq \tilde{\beta}_{-q}\left(g_{2} g_{1}\right)$ as elements of $P G L(2, \mathbb{C})$. The groups $S_{3}, A_{4}$ and $A_{5}$ have only abelian proper quotients and hence $\tilde{\beta}_{-q}$ should be injective for $n \in\{2,3,5\}$. When $n=4$ the element $\tilde{\beta}_{-q}\left(g_{1}\right)$ has order 4 in $P G L(2, \mathbb{C})$ and hence the image of $\tilde{\beta}_{\overline{-}}$ cannot be $S_{3}$. Since $S_{4}$ has only $S_{3}$ and its abelian quotients as proper quotients it follows that $\tilde{\beta}_{-q}$ is also injective when $n=4$.

Alternatively, we can use directly the computations made by Jones in [17]. This implies that $\beta_{-q}$ is injective as well and, in particular, $\beta_{-q}\left(B_{3}\right)$ has the given presentation, establishing the claim.

The two excluded cases which have to be treated separately are as follows:

Proposition 3.2. $\quad$ 1. If $q=1$, then $\beta_{-q}\left(B_{3}\right)$ is the subgroup $S L(2, \mathbb{Z})$ of $G L(2, \mathbb{C})$ with the presentation:

$$
\left\langle g_{1}, g_{2} \mid g_{1} g_{2} g_{1}=g_{2} g_{1} g_{2},\left(g_{1} g_{2}\right)^{6}=1\right\rangle .
$$

2. If $q$ is a primitive 6 -th root of unity, then the representation $\beta_{-q}$ of $B_{3}$ is not completely reducible and its image $\beta_{-q}\left(B_{3}\right)$ has the presentation:

$$
\left\langle g_{1}, g_{2} \mid g_{1} g_{2} g_{1}=g_{2} g_{1} g_{2}, g_{1}^{6}=1, g_{1}^{-2} g_{2}=g_{2} g_{1}^{2}\right\rangle .
$$

Proof. The group $\beta_{-1}\left(B_{3}\right)$ is generated by the images of the generators, namely $\left(\begin{array}{ll}1 & 1 \\ 0 & 1\end{array}\right)$ and $\left(\begin{array}{cc}1 & 0 \\ -1 & 1\end{array}\right)$, and thus it coincides with $S L(2, \mathbb{Z})$ and the presentation follows.

Let $q$ be a primitive 6 -th root of unity, so that $t=-q$ is a primitive third root of unity. Let $V=\left(\begin{array}{cc}-t & 0 \\ 1 & 1\end{array}\right)$. We denote by $\Gamma$ the subgroup $V^{-1} \rho_{t}\left(B_{3}\right) V$ of $G L(2, \mathbb{C})$. Then the matrices $h_{i}=V^{-1} \beta_{t}\left(g_{i}\right) V$ are both upper triangular, namely:

$$
h_{1}=\left(\begin{array}{cc}
1 & -t^{2} \\
0 & -t
\end{array}\right), h_{2}=\left(\begin{array}{cc}
1 & 0 \\
0 & -t
\end{array}\right)
$$

We have therefore:

$$
h_{1} h_{2}^{-1}=\left(\begin{array}{ll}
1 & t \\
0 & 1
\end{array}\right), h_{2}^{-1} h_{1}=\left(\begin{array}{cc}
1 & t+1 \\
0 & 1
\end{array}\right), h_{2} h_{1}^{-1} h_{2}^{-1} h_{1}=\left(\begin{array}{ll}
1 & 1 \\
0 & 1
\end{array}\right) .
$$

Since the diagonal of the generators $h_{i}$ is $(1,-t)$ the group $\Gamma$ is contained in the group of matrices:

$$
\tilde{\Gamma}=\left\{\left(\begin{array}{ll}
1 & r+s t \\
0 & (-t)^{m}
\end{array}\right), m \in \mathbb{Z} / 6 \mathbb{Z}, r, s \in \mathbb{Z}\right\} \subset G L(2, \mathbb{C}) .
$$

Any matrix in $\tilde{\Gamma}$ can be written as a product

$$
h_{2}^{m}\left(h_{1} h_{2}^{-1}\right)^{s}\left(h_{2} h_{1}^{-1} h_{2}^{-1} h_{1}\right)^{r},
$$


such that $\Gamma$ coincides with $\tilde{\Gamma}$.

Observe now that the map $p: \Gamma \rightarrow \mathbb{Z} / 6 \mathbb{Z}$ defined by:

$$
p\left(\begin{array}{ll}
1 & r+s t \\
0 & (-t)^{m}
\end{array}\right)=m \in \mathbb{Z} / 6 \mathbb{Z}
$$

is a well-defined homomorphism. Then we obtain the exact sequence:

$$
1 \rightarrow \mathbb{Z}^{2} \rightarrow \Gamma \rightarrow \mathbb{Z} / 6 \mathbb{Z} \rightarrow 1
$$

where the inclusion $i: \mathbb{Z}^{2} \rightarrow \Gamma$ is given by $i(1,0)=h_{1} h_{2}^{-1}$ and $i(0,1)=h_{2} h_{1}^{-1} h_{2}^{-1} h_{1}$. Thus $\Gamma$ is a polycyclic group. Denote by $u=h_{1} h_{2}^{-1}$ and $v=h_{2} h_{1}^{-1} h_{2}^{-1} h_{1}$ the two generators of the kernel of $p$. We obtain an explicit presentation of $\Gamma$ out of one of $\mathbb{Z}^{2}$ by adding the generator $h_{2}$ of order 6 whose image generates $p(\Gamma)$ and the relations which describe its action by conjugacy on $\mathbb{Z}^{2}$. Specifically, we have:

$$
\Gamma=\left\langle u, v, h_{2} \mid u v=v u, h_{2}^{6}=1, h_{2} u h_{2}^{-1}=v^{-1}, h_{2} v h_{2}^{-1}=u v\right\rangle .
$$

Now, in order to describe $\Gamma$ as a quotient of $B_{3}$ we add the redundant generator $h_{1}$ and the braid relation and express $u, v$ in terms of the $h_{i}$. The conjugacy relations are now consequences of the braid relation while the commutativity relation is equivalent to $h_{2} h_{1}^{2}=h_{1}^{-2} h_{2}$. This gives the desired presentation for the image $\beta_{-q}\left(B_{3}\right)$.

\section{$3.2 \quad$ Discrete subgroups of $P U(1,1)$}

The aim of this section is to find whether the image of Burau's representation $\beta_{-q}$ is a discrete subgroup in $P U(1,1)$. The main result of this section is the identification of the image of a free subgroup of $P B_{3}$ by Burau's representation with a group generated by two rotations. Then some results of Knapp, Mostow and Deraux $([13,19,26])$ give necessary and sufficient conditions for such a subgroup to be discrete.

Instead of an explicit argument we could use below the fact that two irreducible subgroups $G$ and $G^{\prime}$ of $S L(2, \mathbb{C})$ generated by two matrices $A, B$ and $A^{\prime}, B^{\prime}$ respectively are conjugate (by a matrix which conjugates the respective generators) if and only if $\operatorname{tr}(A)=\operatorname{tr}\left(A^{\prime}\right), \operatorname{tr}(B)=\operatorname{tr}\left(B^{\prime}\right)$ and $\operatorname{tr}(A B)=\operatorname{tr}\left(A^{\prime} B^{\prime}\right)$, where tr denotes the trace.

Let us denote by $A=\beta_{-q}\left(g_{1}^{2}\right)$ and $B=\beta_{-q}\left(g_{2}^{2}\right)$ and $C=\beta_{-q}\left(\left(g_{1} g_{2}\right)^{3}\right)$. As is well-known $P B_{3}$ is isomorphic to the direct product $\mathbb{F}_{2} \times \mathbb{Z}$, where $\mathbb{F}_{2}$ is freely generated by $g_{1}^{2}$ and $g_{2}^{2}$ and the factor $\mathbb{Z}$ is the center of $B_{3}$ generated by $\left(g_{1} g_{2}\right)^{3}$.

It is simple to check that:

$$
A=\left(\begin{array}{cc}
q^{2} & 1+q \\
0 & 1
\end{array}\right), B=\left(\begin{array}{cc}
1 & 0 \\
-q-q^{2} & q^{2}
\end{array}\right), C=\left(\begin{array}{cc}
-q^{3} & 0 \\
0 & -q^{3}
\end{array}\right) .
$$

Recall that $P S L(2, \mathbb{Z})$ is the quotient of $B_{3}$ by its center. Since $C$ is a scalar matrix the homomorphism $\beta_{-q}: B_{3} \rightarrow G L(2, \mathbb{C})$ factors to a homomorphism $P S L(2, \mathbb{Z}) \rightarrow P G L(2, \mathbb{C})$.

We will be concerned below with the subgroup $\Gamma_{-q}$ of $P G L(2, \mathbb{C})$ generated by the images of $A$ and $B$ in $P G L(2, \mathbb{C})$. When $\beta_{-q}$ is unitarizable, the group $\Gamma_{-q}$ can be viewed as a subgroup of the pseudo-unitary group $P U(1,1)$. Specifically, consider the action of $\Gamma_{-q}$ on the projective line $\mathbb{C} P^{1}$. Let $V$ be the matrix in the proof of Proposition 2.3, namely: $V=\left(\begin{array}{cc}a & \frac{1}{(1-q) a} \\ 0 & \frac{1}{a}\end{array}\right)$, for $-q \neq-1$, 
where $a$ is given by $(1-q)^{3} C a^{2}=1-q+q^{2}$ and $C \neq 0$ is chosen such that $\rho_{-q, C}$ is unitarizable. Denote the conjugate $V^{-1} Z V$ by $\bar{Z}$. We have then:

$$
\bar{A}=\left(\begin{array}{cc}
q^{2} & 0 \\
0 & 1
\end{array}\right), \bar{B}=\left(\begin{array}{cc}
\frac{1+q^{2}}{1-q} & \frac{1+q^{3}}{(1-q)^{2} a^{2}} \\
-q(1+q) a^{2} & -\frac{q+q^{3}}{1-q}
\end{array}\right), \overline{A B}=\left(\begin{array}{cc}
\frac{q^{2}-q^{4}}{1-q} & \frac{q^{2}+q^{5}}{(1-q)^{2} a^{2}} \\
-q(1+q) a^{2} & -\frac{q+q^{3}}{1-q}
\end{array}\right) .
$$

since $A B=\left(\begin{array}{cc}-q^{3}-q^{2}-q & q^{2}+q^{3} \\ -q-q^{2} & q^{2}\end{array}\right)$.

We know that $V^{-1} \beta_{-q} V=\sigma \otimes \rho_{-q, C}$ and $\sigma \otimes \rho_{-q, C}$ is unitarizable simply by rescaling. In fact $\lambda\left(\sigma \otimes \rho_{-q, C}\right)$ is pseudo-unitary (for those values of $-q$ considered in Proposition 2.1) when $\lambda$ verifies the condition $\lambda^{2} q=1$. Since scalar rescaling does not affect the class of the matrix in $P U(1,1)$ we can work directly with the classes of the matrices $\bar{A}$ and $\bar{B}$ in $P U(1,1)$.

Definition 3.1. Let $q=\exp (i \alpha)$, with $\alpha \in\left(-\frac{\pi}{3}, \frac{\pi}{3}\right)$. The group $\Gamma_{-q} \subset P U(1,1)$ is the subgroup generated by the classes $\beta_{-q}\left(g_{1}^{2}\right)$ and $\beta_{-q}\left(g_{2}^{2}\right)$, namely the classes of matrices $\bar{A}, \bar{B}$ in $P U(1,1)$.

It appears that the search for discrete subgroups in the pseudo-unitary case is more interesting than in the unitary case since we can find infinite discrete subgroups of $P U(1,1)$. The main result in this section is the following:

Proposition 3.3. Let $q=\exp (i \alpha)$, with $\alpha \in\left(-\frac{\pi}{3}, \frac{\pi}{3}\right)$. Then the group $\Gamma_{-q}$ is a discrete subgroup of $P U(1,1)$ if and only if $q=\exp \left(\frac{ \pm 2 \pi i}{n}\right)$, for $n \in \mathbb{Z}_{+}$and $n \geq 7$.

Proof. Recall that $P U(1,1)$ is a subgroup of $P G L(2, \mathbb{C})$ which keeps invariant (and hence acts on) the unit disk $\mathbb{D} \subset \mathbb{C} P^{1}$. The action of $P U(1,1)$ on $\mathbb{D}$ is conjugate to the action of the isomorphic group $\operatorname{PSL}(2, \mathbb{R})$ on the upper half plane. The former is simply the action by isometries on the disk model of the hyperbolic plane.

The key point of our argument is the existence of a fundamental domain for the action of $\Gamma_{-q}$ on $\mathbb{D}$. We will look to the fixed points of the isometries $\bar{A}, \bar{B}, \overline{A B}$ on the hyperbolic disk $\mathbb{D}$. We have the following list:

1. $\bar{A}$ has the fixed point set $\{0, \infty\}$ in $\mathbb{C} P^{1}$, and thus a unique fixed point in $\mathbb{D}$, namely its center $O$.

2. $\bar{B}$ has the fixed point set $\left\{-\frac{1}{(1-q) a^{2}},-\frac{q^{2}-q+1}{q(1-q) a^{2}}\right\} \subset \mathbb{C} P^{1}$ and thus a unique fixed point in $\mathbb{D}$, namely $P=-\frac{q^{2}-q+1}{q(1-q) a^{2}}$. In fact, if $\cos (\alpha+\pi) \in\left[-1,-\frac{1}{2}\right]$, then

$$
\left|\frac{q^{2}-q+1}{q(1-q) a^{2}}\right|=|1-q||a|^{2}=\sqrt{\left|1-q+q^{2}\right|}=\sqrt{1+2 \cos (\alpha+\pi)} \in[0,1] .
$$

3. $\overline{A B}$ has the fixed point set $\left\{-\frac{q}{(1-q) a^{2}},-\frac{q^{2}-q+1}{(1-q) a^{2}}\right\} \subset \mathbb{C} P^{1}$ and thus a unique fixed point in $\mathbb{D}$, namely $Q=-\frac{q^{2}-q+1}{(1-q) a^{2}}$.

We have now the following lemma, whose proof is postponed a few lines later:

Lemma 3.1. The elements $\bar{A}, \bar{B}$ and $\overline{A B}$ of $P U(1,1)$ are rotations of the same angle $2 \alpha$ centered at the three vertices of the equilateral geodesic triangle $\Delta=O P Q$ in the hyperbolic plane $\mathbb{D}$, whose angles are equal to $\alpha$.

Eventually we state the following result of Knapp from [19], later rediscovered by Mostow (see [26]) and Deraux ([13], Theorem 7.1): 
Lemma 3.2. The three rotations of angle $2 \alpha$ in the hyperbolic plane $\mathbb{D}$ around the vertices of an equilateral hyperbolic triangle $\Delta$ of angles $\alpha>0$ generate a discrete subgroup of $P U(1,1)$ if and only if $\alpha=\frac{2 \pi}{n}$, with $n \in \mathbb{Z}_{+}$and $n \geq 7$.

Notice that the existence of a hyperbolic triangle of angles equal to $\alpha$ requires that $n \geq 7$.

The two lemmas from above yield the result claimed in Proposition 3.3.

Proof of Lemma 3.1. We know from above that $\bar{A}, \bar{B}$ and $\overline{A B}$ are elliptic elements of $P U(1,1)$. Actually all of them are rotations of angle $\pm 2 \alpha$ :

1. $\bar{A}(z)=q^{2} z$ and hence $\bar{A}$ is the counterclockwise rotation of angle $2 \alpha$ around $O$;

2. $\bar{B}$ is conjugate to $\bar{A}$ and thus is a rotation of angle $\pm 2 \alpha$ around $P$;

3. $\overline{A B}$ has the eigenvalues $-q^{3}$ and $-q$, which are distinct since $q^{2} \neq 1$, and so is diagonalizable. Therefore $\overline{A B}$ is a rotation of angle $\pm 2 \alpha$ around $Q$.

Consider now the geodesic triangle $\Delta=O P Q$ in $\mathbb{D}$. The angle $\widehat{P O Q}$ at $O$ equals $\alpha$ since $Q=q P$. Since the argument of $q$ is acute it follows that the orientation of the arc $P Q$ is counterclockwise. Moreover, this shows that $d(O, P)=d(O, Q)$, where $d$ denotes the hyperbolic distance in $\mathbb{D}$ and hence we obtain the equality of angles $\widehat{O P Q}=\widehat{O Q P}$.

Let us introduce the element $D=\beta_{-q}\left(g_{2}\right)$, which verifies $D^{2}=B$. Then $\bar{D}^{2}=\bar{B}$. We can compute

$$
\bar{D}=\left(\begin{array}{cc}
\frac{1}{1-q} & \frac{q^{2}-q+1}{(1-q)^{2} a^{2}} \\
-q a^{2} & \frac{q^{2}}{1-q}
\end{array}\right) .
$$

We know that $\bar{D}$ is a rotation of angle $\pm \alpha$ around $P$ since is conjugated to $\beta_{-q}\left(g_{1}\right)$. We can check that $\bar{D}(Q)=0$ and hence $\bar{D}$ is the counterclockwise rotation of angle $\alpha$ around $P$ and $d(P, Q)=d(P, O)$. Thus all angles of the triangle $\Delta$ are equal to $\alpha$. This also shows that $\bar{B}$ is the counterclockwise rotation of angle $2 \alpha$.

Since both $\bar{A}$ and $\bar{B}$ are counterclockwise rotations of angle $2 \alpha$ it follows that $\overline{A B}$ is also the counterclockwise rotation of angle $2 \alpha$.

\subsection{Triangle groups as images of a free pure braid subgroup}

The aim of this section is to obtain finite presentations for the groups $\Gamma_{-q}$. Discrete subgroups of $P U(1,1)$ could be given explicit presentations if we are able to find a fundamental domain for their action on the hyperbolic disk $\mathbb{D}$. This method goes back to Poincaré and we refer to [3] for more details. We find that, when discrete, $\Gamma_{-q}$ is a suitable triangle group and thus we have an obvious presentation of it.

Before we proceed we make a short digression on triangle groups. Let $\Delta$ be a geodesic triangle in the hyperbolic plane of angles $\frac{\pi}{m}, \frac{\pi}{n}, \frac{\pi}{p}$, so that $\frac{1}{m}+\frac{1}{n}+\frac{1}{p}<1$. The extended triangle group $\Delta^{*}(m, n, p)$ is the group of isometries of the hyperbolic plane generated by the three reflections $R_{1}, R_{2}, R_{3}$ with respect to the edges of $\Delta$. It is well-known that a presentation of $\Delta^{*}(m, n, p)$ is given by

$$
\Delta^{*}(m, n, p)=\left\langle R_{1}, R_{2}, R_{3} ; R_{1}^{2}=R_{2}^{2}=R_{3}^{2}=1,\left(R_{1} R_{2}\right)^{m}=\left(R_{2} R_{3}\right)^{n}=\left(R_{3} R_{1}\right)^{p}=1\right\rangle .
$$

The second type of relations have a simple geometric meaning. In fact, the product of the reflections with respect to two adjacent edges is a rotation by the angle which is twice the angle between those 
edges. The subgroup $\Delta(m, n, p)$ generated by the rotations $a=R_{1} R_{2}, b=R_{2} R_{3}, c=R_{3} R_{1}$ is a normal subgroup of index 2 , which coincides with the subgroup of isometries preserving the orientation. One calls $\Delta(m, n, p)$ the triangle (also called triangular, or von Dyck) group associated to $\Delta$. Moreover, the triangle group has the presentation:

$$
\Delta(m, n, p)=\left\langle a, b, c ; a^{m}=b^{n}=c^{p}=1, a b c=1\right\rangle .
$$

Observe that $\Delta(m, n, p)$ also makes sense when $m, n$ or $p$ are negative integers, by interpreting the associated generators as clockwise rotations. The triangle $\Delta$ is a fundamental domain (see [3]) for the action of $\Delta^{*}(m, n, p)$ on the hyperbolic plane. Thus a fundamental domain for $\Delta(m, n, p)$ consists of the union $\Delta^{*}$ of $\Delta$ with the reflection of $\Delta$ in one of its edges.

Proposition 3.4. Let $m<k$ be such that $\operatorname{gcd}(m, k)=1$ where $k \geq 4$. Then the group $\Gamma_{-\exp }\left(\frac{ \pm 2 m \pi i}{2 k}\right)$ is a triangle group with the presentation:

$$
\Gamma_{-\exp \left(\frac{ \pm 2 m \pi i}{2 k}\right)}=\left\langle A, B ; A^{k}=B^{k}=(A B)^{k}=1\right\rangle .
$$

Proof. Denote by $\Delta\left(\frac{\pi}{\alpha}, \frac{\pi}{\alpha}, \frac{\pi}{\alpha}\right)$ the group generated by the rotations of angle $2 \alpha$ around vertices of the triangle $\Delta$ of angles $\alpha$. We will use this notation even when $\alpha$ is not an integral part of $\pi$ i.e., $\alpha$ cannot be written as $\frac{\pi}{k}$, with $k \in \mathbb{Z}$. We saw above that $\Gamma_{-q}$ is isomorphic to $\Delta\left(\frac{\pi}{\alpha}, \frac{\pi}{\alpha}, \frac{\pi}{\alpha}\right)$.

When $\alpha=\frac{2 \pi}{2 k}$, the group $\Delta\left(\frac{\pi}{\alpha}, \frac{\pi}{\alpha}, \frac{\pi}{\alpha}\right)$ is a triangle group, namely it has the rhombus $\Delta^{*}$ as a fundamental domain for its action on $\mathbb{D}$. In particular, $\Gamma_{-q}$ is the triangle group with the given presentation.

For the general case of $\alpha=\frac{2 \pi m}{2 k}$ where $q$ is a primitive $2 k$-th root of unity the situation is however quite similar. There is a Galois conjugation sending $-q$ into $-\exp \left(\frac{ \pm 2 \pi i}{2 k}\right)$, which induces an automorphism of $P G L(2, \mathbb{C})$. Although this automorphism does not preserve the discreteness it is an isomorphism of $\Gamma_{-q}$ onto $\Gamma_{-\exp \left(\frac{ \pm 2 \pi i}{2 k}\right)}$. This settles the claim.

If $n$ is odd $n=2 k+1$, then the group $\Gamma_{-q}$ is a quotient of the triangle group associated to $\Delta$, which embeds into the group associated to some sub-triangle $\Delta^{\prime}$ of $\Delta$. Before to proceed with the odd case we need the following:

Lemma 3.3. The natural embedding of $\Delta\left(\frac{2 k+1}{2}, \frac{2 k+1}{2}, \frac{2 k+1}{2}\right)$ into $\Delta(2,3,2 k+1)$ is an isomorphism.

Proof. A simple geometric computation shows that:

$$
a=\alpha^{2}, b=v \alpha^{2} v=u^{2} \alpha^{2} u, c=u \alpha^{2} u^{2} .
$$

Therefore $\alpha=a^{k+1} \in \Delta\left(\frac{2 k+1}{2}, \frac{2 k+1}{2}, \frac{2 k+1}{2}\right)$.

From the relation $\alpha u v=1$ we derive $a^{k+1} u v=1$, and thus $u=a^{k} v$. The relation $u^{3}=1$ reads now $a^{k}\left(v a^{k} v\right) a^{k} v=1$ and replacing $b^{k}$ by $v a^{k} v$ we find that $v=a^{k} b^{k} a^{k} \in \Delta\left(\frac{2 k+1}{2}, \frac{2 k+1}{2}, \frac{2 k+1}{2}\right)$.

Further $u=a^{k} v=a^{-1} b^{k} a^{k} \in \Delta\left(\frac{2 k+1}{2}, \frac{2 k+1}{2}, \frac{2 k+1}{2}\right)$. This means that $\Delta\left(\frac{2 k+1}{2}, \frac{2 k+1}{2}, \frac{2 k+1}{2}\right)$ is actually $\Delta(2,3,2 k+1)$, as claimed.

Proposition 3.5. Let $0<m<2 k+1$ be such that $\operatorname{gcd}(m, 2 k+1)=1$ and $k \geq 3$. Then the group $\Gamma_{-\exp \left(\frac{ \pm 2 m \pi i}{2 k+1}\right)}$ is isomorphic to the triangle group $\Delta(2,3,2 k+1)$ and has the following presentation (in terms of our generators $A, B$ ):

$$
\Gamma_{-\exp \left(\frac{ \pm 2 m \pi i}{2 k+1}\right)}=\left\langle A, B ; A^{2 k+1}=B^{2 k+1}=(A B)^{2 k+1}=1,\left(A^{-1} B^{k}\right)^{2}=1,\left(B^{k} A^{k-1}\right)^{3}=1\right\rangle .
$$


Proof. It suffices to consider the case $m=1$, as in the previous Proposition. The proof of the discreteness in ([13], Theorem 7.1) shows that the group $\Delta\left(\frac{2 k+1}{2}, \frac{2 k+1}{2}, \frac{2 k+1}{2}\right)$, which is generated by the rotations $a, b, c$ around the vertices of the triangle $\Delta$ embeds into the triangle group associated to a smaller triangle $\Delta^{\prime}$. One constructs $\Delta^{\prime}$ by considering all geodesics of $\Delta$ joining a vertex and the midpoint of its opposite side. The three median geodesics pass through the barycenter of $\Delta$ and subdivide $\Delta$ into 6 equal triangles. We can take for $\Delta^{\prime}$ any one of the 6 triangles of the subdivision. It is immediate that $\Delta^{\prime}$ has angles $\frac{\pi}{2 k+1}, \frac{\pi}{2}$ and $\frac{\pi}{3}$ so that the associated triangle group is $\Delta(2,3,2 k+1)$. This group has the presentation:

$$
\Delta(2,3,2 k+1)=\left\langle\alpha, u, v ; \alpha^{2 k+1}=u^{3}=v^{2}=\alpha u v=1\right\rangle,
$$

where the generators are the rotations of double angle around the vertices of the triangle $\Delta^{\prime}$.

Now, Lemma 3.3 shows that it suffices to find a presentation of $\Delta(2,3,2 k+1)$ that uses the generators $A=a, B=b$. It is not difficult to show that the group with the presentation of the statement is isomorphic to $\Delta(2,3,2 k+1)$, the inverse homomorphism sending $\alpha$ into $A^{k+1}, u$ into $A^{-1} B^{k} A^{k}$ and $v$ into $A^{k} B^{k} A^{k}$.

A direct consequence of Propositions 3.4 and 3.5 is the following abstract description of the image of Burau's representation:

Corollary 3.1. If $q$ is a primitive root of unity whose order is not in the set $\{1,2,3,4,6,10\}$, then $\Gamma_{q}$ is an infinite triangle group.

Alternatively, we obtain a set of normal generators for the kernel of Burau's representation, as follows:

Corollary 3.2. Let $n \notin\{1,2,3,4,5,6\}$ and $q$ a primitive root of unity of order $n$. We denote by $N(G)$ the normal closure of a subgroup $G$ of $\left\langle g_{1}^{2}, g_{2}^{2}\right\rangle \subset B_{3}$. Then the kernel $\operatorname{ker} \beta_{-q}$ : $\left\langle g_{1}^{2}, g_{2}^{2}\right\rangle \rightarrow P G L(2, \mathbb{C})$ of the restriction of Burau's representation is given by:

$$
\begin{cases}N\left(\left\langle g_{1}^{2 k}, g_{2}^{2 k},\left(g_{1}^{2} g_{2}^{2}\right)^{k}\right\rangle\right), & \text { if } n=2 k ; \\ N\left(\left\langle g_{1}^{2(2 k+1)}, g_{2}^{2(2 k+1)},\left(g_{1}^{2} g_{2}^{2}\right)^{2 k+1},\left(g_{1}^{-2} g_{2}^{2 k}\right)^{2},\left(g_{2}^{2 k} g_{1}^{2(k-1)}\right)^{3}\right\rangle\right), & \text { if } n=2 k+1 .\end{cases}
$$

\subsection{Proof of Theorem 1.2}

In order to prove Theorem 1.2 we need some preliminary lemmas explaining how to retrieve the kernel of Burau's representation of $B_{3}$ from known information on its restriction to the free subgroup $\left\langle g_{1}^{2}, g_{2}^{2}\right\rangle$ of $P B_{3}$.

The case when $q$ is of odd order is particularly simple:

Lemma 3.4. If $n=2 k+1, k \geq 3$, the inclusion $P B_{3} \subset B_{3}$ induces an isomorphism:

$$
\frac{P B_{3}}{P B_{3} \cap \operatorname{ker} \beta_{-q}} \rightarrow \frac{B_{3}}{\operatorname{ker} \beta_{-q}} .
$$

Equivalently, we have an exact sequence:

$$
1 \rightarrow P B_{3} \cap \operatorname{ker} \beta_{-q} \rightarrow \operatorname{ker} \beta_{-q} \rightarrow S_{3} \rightarrow 1 .
$$

Proof. The induced map is clearly an injection. Observe next that $g_{1}^{2 k+1}, g_{2}^{2 k+1} \in \operatorname{ker} \beta_{-q}$ and thus for every $x \in B_{3}$ there exists some $\eta \in \operatorname{ker} \beta_{-q}$ such that $\eta x \in P B_{3}$. Thus the image of the class $\eta x$ is the class of $x$ and this shows that the induced homomorphism is also surjective. The claims follow. 
When $q$ has an even order we will need an additional combinatorial argument:

Lemma 3.5. If $n=2 k, k \geq 4$, then $\operatorname{ker} \beta_{-q} \subset P B_{3}$. Thus the inclusion $P B_{3} \subset B_{3}$ induces the exact sequence:

$$
1 \rightarrow \frac{P B_{3}}{P B_{3} \cap \operatorname{ker} \beta_{-q}} \rightarrow \frac{B_{3}}{\operatorname{ker} \beta_{-q}} \rightarrow S_{3} \rightarrow 1
$$

Proof. It suffices to show that $\beta_{-q}(g) \notin \beta_{-q}\left(P B_{3}\right)$ for $g \in\left\{g_{1}, g_{2}, g_{1} g_{2}, g_{2} g_{1}, g_{1} g_{2} g_{1}\right\}$. Since none of $\beta_{-q}(g)$, for $g$ as above is a scalar matrix, this claim is equivalent to show that $\beta_{-q}(g) \notin$ $\beta_{-q}\left(\left\langle g_{1}^{2}, g_{2}^{2}\right\rangle\right)=\langle A, B\rangle$. We will conjugate everything and work instead with $\bar{A}$ and $\bar{B}$. The triangle group generated by $\bar{A}$ and $\bar{B}$ has a fundamental domain consisting of the rhombus $\Delta^{*}$, which is the union of $\Delta$ with its reflection image $R_{j} \Delta$. The common edge of the two triangles of the rhombus will be called a diagonal.

The image of $g_{i}$ is the rotation of angle $\alpha$ around a vertex of the triangle $\Delta$. If this rotation were an element of $\Delta(k, k, k)$, then it would act as an automorphism of the tessellation with copies of $\Delta^{*}$. When the vertex fixed by $g_{i}$ lies on the diagonal of $\Delta^{*}$, then a rotation of angle $\alpha$ sends the rhombus onto an overlapping rhombus (having one triangle in common) and thus it cannot be an automorphism of the tessellation, which is a contradiction.

This argument does not work when the vertex is opposite to the diagonal. However, let us color the triangle $\Delta$ in white and $R_{j} \Delta$ in black. Continue this way by coloring all triangles in black and white so that adjacent triangles have different colors. It is easy to see that the rotations of angle $2 \alpha$ (and hence all elements of the group $\Delta(k, k, k)$ ) send white triangles into white triangles. But the rotation of angle $\alpha$ around a vertex opposite to the diagonal sends a white triangle into a black one. This contradiction shows that the image of the $g_{i}$ does not belong to $\Delta(k, k, k)$.

The last cases are quite similar. The images of $g_{1} g_{2}$ and $g_{2} g_{1}$ send $\Delta^{*}$ into an overlapping rhombus having one triangle in common. Eventually the image of $g_{1} g_{2} g_{1}$ does not preserve the black and white coloring. This proves the lemma.

We are now able to prove Theorem 1.2, which we restate here for the reader's convenience:

Theorem 3.3. Assume that $q$ is a primitive $n$-th root of unity, $n \geq 7$ and $g_{1}, g_{2}$ are the standard generators of $B_{3}$. Then $\beta_{-q}\left(B_{3}\right)$ has a presentation with generators $g_{1}, g_{2}$ and relations:

1. The case $n=2 k$ and $k$ is odd:

$$
\begin{array}{ll}
\text { Braid relation: } & g_{1} g_{2} g_{1}=g_{2} g_{1} g_{2}, \\
\text { Power relations: } & g_{1}^{2 k}=g_{2}^{2 k}=\left(g_{1}^{2} g_{2}^{2}\right)^{k}=1 .
\end{array}
$$

2. The case $n=2 k$ and $k$ is even:

$$
\begin{array}{ll}
\text { Braid relation: } & g_{1} g_{2} g_{1}=g_{2} g_{1} g_{2}, \\
\text { Power relations: } & g_{1}^{2 k}=g_{2}^{2 k}=\left(g_{1}^{2} g_{2}^{2}\right)^{2 k}=1 .
\end{array}
$$

3. The case $n=2 k+1$ :

$$
\begin{array}{ll}
\text { Braid relation: } & g_{1} g_{2} g_{1}=g_{2} g_{1} g_{2}, \\
\text { Power relations: } & g_{1}^{2 k+1}=g_{2}^{2 k+1}=\left(g_{1}^{2} g_{2}^{2}\right)^{2(2 k+1)}=1 .
\end{array}
$$

Proof. The strategy of the proof is to lift the triangle group presentation of $\Gamma_{-q}$ to $\beta_{-q}\left(\left\langle g_{1}^{2}, g_{2}^{2}\right\rangle\right)$ and then to $\beta_{-q}\left(P B_{3}\right)$, by adding a central generator. We add further the standard generators $g_{1}, g_{2}$ of $B_{3}$ and use the previous two lemmas in order to obtain a presentation of $\beta_{-q}\left(B_{3}\right)$ and then get rid of redundant generators and relations. 
Lemma 3.5 shows that $\operatorname{ker} \beta_{-q}$ has the same normal generators as $\operatorname{ker} \beta_{-q} \cap P B_{3}$, when $n$ is even. Lemma 3.4 states that for odd $n=2 k+1$ a set of normal generators of ker $\beta_{-q}$ is obtained by adding the two elements $g_{1}^{2 k+1}$ and $g_{2}^{2 k+1}$ to a set of normal generators of ker $\beta_{-q} \cap P B_{3}$. In this way one produces a presentation of $\beta_{-q}\left(B_{3}\right)$ from a presentation of $\beta_{-q}\left(P B_{3}\right)$.

Furthermore, $P B_{3}$ is the direct product of the free group $\left\langle g_{1}^{2}, g_{2}^{2}\right\rangle$ with the center of $B_{3}$, which is generated by $\left(g_{1} g_{2}\right)^{3}$. Now $\beta_{-q}\left(g_{1} g_{2}\right)^{3}$ is the scalar matrix $-q^{3} \mathbf{1}$. The order of $-q^{3}$ is $2 k /(\operatorname{gcd}(3, k) \operatorname{gcd}(2, k+$ 1)) if $q$ is a primitive $2 k$-th root of unity and is equal to $2(2 k+1) / \operatorname{gcd}(3,2 k+1)$ when $q$ is a primitive root of unity of order $2 k+1$. Therefore a presentation of $\beta_{-q}\left(P B_{3}\right)$ can be obtained from a presentation of $\beta_{-q}\left(\left\langle g_{1}^{2}, g_{2}^{2}\right\rangle\right)$ by adjoining a new central generator $\left(g_{1} g_{2}\right)^{3}$ and the following center relations:

$$
\begin{aligned}
& \left(g_{1} g_{2}\right)^{6 k /(\operatorname{gcd}(2, k+1) \operatorname{gcd}(3, k))}=1, \text { for even } n=2 k, \\
& \left(g_{1} g_{2}\right)^{6(2 k+1) / \operatorname{gcd}(3,2 k+1)}=1, \text { for odd } n=2 k+1 .
\end{aligned}
$$

This new central generator will be redundant as soon as we pass to $B_{3}$ with its standard generators $g_{1}, g_{2}$.

The group $\beta_{-q}\left(\left\langle g_{1}^{2}, g_{2}^{2}\right\rangle\right) \subset G L(2, \mathbb{C})$ is a central extension of its image mod scalars $\Gamma_{-q} \subset P G L(2, \mathbb{C})$. Thus we can obtain a presentation of it by looking at the lifts of the relations holding in $\Gamma_{-q}$.

Let $n=2 k$. The lifts of the relations $A^{k}=B^{k}=1$ in $\Gamma_{-q}$ are the relations $g_{1}^{2 k}=g_{2}^{2 k}=1$ in $\beta_{-q}\left(\left\langle g_{1}^{2}, g_{2}^{2}\right\rangle\right)$. The eigenvalues of the matrix $A B$ are $-q^{3}$ and $-q$ so that

$$
\beta_{-q}\left(\left(g_{1}^{2} g_{2}^{2}\right)^{k}\right)= \begin{cases}-\mathbf{1}, & \text { if } k \equiv 0(\bmod 2) \\ \mathbf{1}, & \text { if } k \equiv 1(\bmod 2) .\end{cases}
$$

Thus for odd $k$ it is enough to add the relation $\left(g_{1}^{2} g_{2}^{2}\right)^{k}=1$.

For even $k$ the element $\left(g_{1}^{2} g_{2}^{2}\right)^{k}$ is central of order 2 . On the other hand, one proves by recurrence on $m$ that the following combined relation holds true in $B_{3}$ :

$$
\left(g_{1} g_{2}\right)^{3 m}=g_{1}^{2 m} g_{2}\left(g_{1}^{2} g_{2}^{2}\right)^{m} g_{2}^{-1} .
$$

Taking $m=k$ and recalling that $\left(g_{1} g_{2}\right)^{3}$ is central we find that $g_{1}^{2 k}=1$ implies that:

$$
\left(g_{1}^{2} g_{2}^{2}\right)^{k}=\left(g_{1} g_{2}\right)^{3 k} .
$$

Thus the fact that $\left(g_{1}^{2} g_{2}^{2}\right)^{k}$ is central is a consequence of the braid and power relations. Thus it suffices to add the power relation $\left(g_{1}^{2} g_{2}^{2}\right)^{2 k}=1$, in order to get a presentation of $\beta_{-q}\left(B_{3}\right)$.

For odd $n=2 k+1$ the lifts of the relations $A^{n}=B^{n}=1$ are $g_{1}^{2 n}=g_{2}^{2 n}=1$, which are consequences of the power relations $g_{1}^{n}=g_{2}^{n}=1$. Furthermore, we verify that:

$$
\beta_{-q}\left(\left(g_{1}^{2} g_{2}^{2}\right)^{2 k+1}\right)=-\mathbf{1},
$$

hence $\left(g_{1}^{2} g_{2}^{2}\right)^{2 k+1}$ is central of order 2 . The argument used above for even $k$ shows that $g_{1}^{2 k+1}=1$ and the braid relations imply that $\left(g_{1}^{2} g_{2}^{2}\right)^{2 k+1}$ is central, so it suffices to add the last power relation $\left(g_{1}^{2} g_{2}^{2}\right)^{2(2 k+1)}=1$. The remaining lifts of relations in $\Gamma_{-q}$ are redundant. In fact, braid and powers relations give us:

$$
\begin{gathered}
\left(g_{1}^{-2} g_{2}^{2 k}\right)^{2}=\left(g_{1}^{-2} g_{2}^{-1}\right)^{2}=\left(g_{1} g_{2}\right)^{-3}, \\
\left(g_{1}^{2 k} g_{2}^{2 k-2}\right)^{3}=\left(g_{1}^{-1} g_{2}^{-3}\right)^{3}=\left(g_{1} g_{2}\right)^{-6} .
\end{gathered}
$$

Eventually, a direct inspection shows that center relations are obtained from the combined relation above along with the braid and power relations. 


\section{References}

[1] J. E. Andersen, Asymptotic faithfulness of the quantum $\mathrm{SU}(n)$ representations of the mapping class groups, Ann. of Math. 163(2006), 347-368.

[2] J. E. Andersen, G. Masbaum and K. Ueno, Topological Quantum Field Theory and the Nielsen-Thurston classification of $M(0,4)$, Math. Proc. Cambridge Phil. Soc. 141(2006), 477-488.

[3] A. F. Beardon, The geometry of discrete groups, Corrected reprint of the 1983 original. Graduate Texts in Mathematics, 91, Springer-Verlag, New York, 1995.

[4] S. Bigelow, The Burau representation is not faithful for $n=5$, Geom. Topol. 3(1999), 397-404.

[5] J. Birman, Braids, Links, and Mapping Class Groups, Ann. Math. Studies, Princeton Univ. Press, 1969.

[6] C. Blanchet, N. Habegger, G. Masbaum and P. Vogel, Topological quantum field theories derived from the Kauffman bracket, Topology 34(1995), 883-927.

[7] T. Church and B. Farb, Infinite generation of the kernels of the Magnus and Burau representations, Algebr. Geom. Topol. 10 (2010), 837-851.

[8] D. Cooper and D. D. Long, A presentation for the image of Burau(4) $\otimes \mathbb{Z}_{2}$, Invent. Math. 127(1997), $535-570$.

[9] A. Coste and T. Gannon, Congruence subgroups and rational conformal field theory, arxiv:math/9909080.

[10] H. S. M. Coxeter: On factors of braid groups, Proc.4-th Canadian Math.Congress, Banff 1957, Univ.Toronto Press 1959, p. 95-122.

[11] H. S. M. Coxeter: Regular complex polytopes, Cambridge Univ. Press 1974.

[12] H. S. M. Coxeter and W. O. J. Moser, Generators and relations for discrete groups. Fourth edition, Ergebnisse der Mathematik und ihrer Grenzgebiete, 14, Springer-Verlag, Berlin-New York, 1980. ix+169 pp.

[13] M. Deraux, On the universal cover of certain exotic Kähler surfaces of negative curvature, Math. Annalen 329(2004), 653-683.

[14] M. H. Freedman, K. Walker and Z. Wang, Quantum SU(2) faithfully detects mapping class groups modulo center, Geom. Topol. 6(2002), 523-539.

[15] L. Funar and T. Kohno, Free subgroups within the images of quantum representations, 19p., Forum Math., to appear, arxiv:1108.4904.

[16] P. Gilmer and G. Masbaum, Integral TQFT for a one-holed torus, Pacific J. Math. 252(2011), 93-112.

[17] V. F. R. Jones, Braid groups, Hecke algebras and type $I_{1}$ factors, in Geometric methods in operator algebras, Proc. US-Japan Seminar, (H. Araki, E. G. Effros Eds.), 1986, 242-271.

[18] F. Klein, Lectures on the icosahedron and the solution of equation of the fifth degree, Dover Publications, 2nd edition (1956) originally published in 1884 .

[19] A. W. Knapp, Doubly generated Fuchsian groups, Michigan J. Math. 15(1968), 289-304.

[20] G. Kuperberg, Denseness and Zariski denseness of Jones braid representations, Geom. Topol. 15(2011), $11-39$.

[21] M. Larsen and Zhenghan Wang, Density of the SO(3) TQFT representation of mapping class groups, Comm. Math. Phys. 260(2005), 641-658.

[22] D. D. Long and M. Paton, The Burau representation is not faithful for $n \geq 6$, Topology 32(1993), 439-447.

[23] G. Masbaum, On representations of mapping class groups in integral TQFT, Oberwolfach Reports, Vol. 5, issue 2, 2008, pp. 1202-1205.

[24] C. T. McMullen, Braid groups and Hodge theory, 2009, Math. Annalen, to appear. 
[25] J. A. Moody, The faithfulness question for the Burau representation, Proc. Amer. Math. Soc. 119(1993), no. 2, 671-679.

[26] G. D. Mostow, On discontinuous action of monodromy groups on the complex n-balls, J. Amer. Math. Soc. 1(1988), 555-586.

[27] L. Paris and D. Rolfsen, Geometric subgroups of mapping class groups, J. Reine Angew. Math. 521(2000), 47-83.

[28] C. Radin and L. Sadun, On 2-generator subgroups of SO(3), Trans. Amer. Math. Soc. 351(1999), 44694480.

[29] R. Santharoubane, Limits of the quantum SO(3) representations for the one-holed torus, arXiv:1202.1813.

[30] C. Squier, The Burau representation is unitary, Proc. Amer. Math. Soc. 90(1984), 199-202.

[31] H. Wenzl, On sequences of projections, C.R.Math. Rep. Acad. Sci. Canada 9(1987), 5-9. 